\title{
Time as an Aid to Improving Security in Smart Cards
}

\author{
Vincent Cordonnier, Professor \\ University of Sciences and Technologies of Lille, France
}

\author{
Anthony Watson, Professor \\ Edith Cowan University, Perth, Australia
}

\author{
Sergiy Nemchenko, Researcher \\ University of Sciences and Technologies of Lille, France
}

Key words: Smart card, security, time logic, application, clock, time slice, operating system.

Abstract: Very often, time is an important function of smart cards applications, particularly for security and authentication. The time data is made available to the program within the card from an external source, namely the application terminal. Unfortunately the card cannot independently authenticate the time data provided by the card terminal and hence the time function is susceptible to corruption by either accidental failure or deliberate fraud introduced via the terminal. Consequently most of the application designers do not consider time as a trusted reference for improving either the flexibility of the application or the level of security. Now, because of technical progress it seems possible to produce time from a clock that is embedded in the card as a part of the silicon chip. This clock in permanently active but the value of time it produces can only be used by the card when it is plugged in to a terminal for a transaction. But usually, this is the only circumstance for a time value to be required by the internal program. Verified 'card time' is then available to any application with different presentations.

The paper first introduces the most significant definitions used for modeling time, such as: event and time slice plus a few elementary notions of temporal 
logic to produce time functions. The physical clock is briefly described as a binary counter where most of the time management is performed either at the operating system level or at the application level. The available time functions are described as dates, delay or cycles.

By using these time functions as guarded commands acting as logical conditions on the regular commands of the card, it becomes possible to offer the application designer options to introduce new security controls

\section{INTRODUCTION}

Generally speaking time issues have been of limited importance for security, other than time stamping, and for smart cards in particular because the card traditionally does not have a time clock. However there are possible alternatives to significantly improve the level of security and give the applications more flexibility by using time constraints and time management in conjunction with other security features of the card. One of the major goals of the present research is to propose a time management tool which a card can use to improve its security function by either restricting the capacity for an attack or preventing such an attack by enabling the card to relate external commands to some time based security conditions. Time control may be considered as an efficient way to limit any fraudulent access. The most significant assertion works at the application level and is based on the proposition that there exists a difference of time utilisation for the transaction and associated management between regular users and fraudulent activities.

Time related factors of smart card use:

a) With an onboard clock a right or privileged activity could have a time limit that can be controlled by the card itself.

A fraud needs more time than the average legitimate use to be completed.

A fraud is often performed when access is not necessary for regular users (for example by night, or out of normal office hours).

A fraudulent access will be likely to use many more commands or make many more access requests during a given time (Attempts to present a key, to open a file or to initiate an application).

The average time for a transaction or a part of transaction may well be different for an attempted fraud (hesitation, consulting a document, etc...)

There may exist some cross relation between time and location of the terminal (application) that can be verified if necessary.( Who is supposed to need access to the office at two AM?).

The date may be used as complementary information for signing a transaction. 
At the operating system level, a clock can be used as a simple and secure tool against time attacks.

One of the main reasons for currently not using the concept of time in smart cards as a security measure is that they do not have any possible internal time reference as commercial versions do not contain a clock. This paper discusses some possibilities for cards to access a time reference provided by either an internal or an external source. Obviously an internal source with the same tamper resistance as the main silicon component would be preferred.

The paper first introduces some formal definitions of temporal logic to identify the kind of tools that are relevant for the purpose. Then a generalised model is proposed for the time security management in a smart card. As time can be used in a different manner for different applications, we discuss the way time references must be provided at the operating system level, according to the requirement of the applications. Then we classify the possible families of time security management assuming that these functions must be a part of the operating system of the card and can be made available to the applications.

In this paper time includes date as well as hours, minutes and seconds and various combinations of this data.

\section{THE TIME MODEL : DEFINITIONS AND FUNCTIONS.}

It is necessary to define the terms used throughout the paper. The basic unit of time is 'the second'.

EVENT : Assuming that time is a continuous flow from the past to the future, an event is a point on that flow. An event is supposed to have a duration equal to zero. It separates the time flow into two subsets, its Past : $\mathbf{P}$ and its Future : F. For example the commencement of a transaction is an event and could be associated with a particular time.

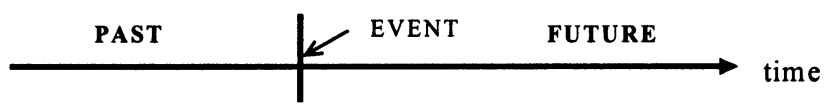

Figure 1. Event

CLOCK : A clock is a source producing the same event on a cyclical basis. The time between two events is constant but that can only be verified by using another clock. 
TIME VARIABLE : A time variable is a Boolean value that can be either TRUE or FALSE according to its relation to one or more event(s).

There are two basic time variables immediately related to any event EV:

PAST(EV) or P(EV) is true for and only for any event belonging to the past of EV.

FUTURE (EV) or F(EV) is true for and only for any event belonging to the future of $\mathrm{EV}$

TIME SLICE: A time slice or DELAY is the amount of time defined between two events.

SECONDARY CLOCKS: They count the number of events or pulses produced by a clock and are able to deliver cyclic slices of time between two of these events.

For example one given day, let us say Wednesday or day3, out of a week clock.

FUNCTION : A time function is any function using Boolean relations between time variables, other time functions or events. Here, it will be used to produce time slices.

Example : Observe two events, EV1 and EV2, where EV2 belongs to the future of EV1.

A time slice function $T$ is only true between these two events :

$T=F(E V 1)$ and $P(E V 2)$

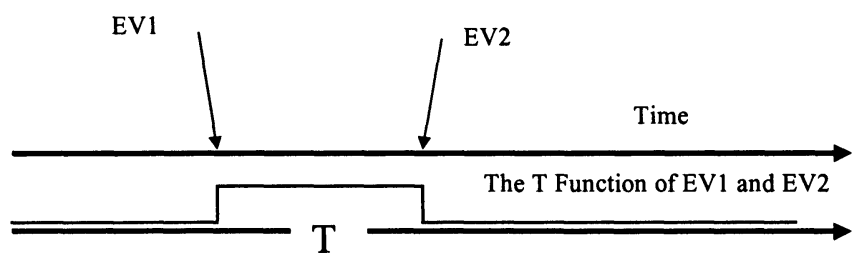

Figure 2. Exemple

\section{HOW TO RELATE TIME AND SECURITY IN A SMART CARD}

A general approach to the problem of time control is to look at any operational function $\mathrm{F}$ which a card can execute and to add some time functions $\mathrm{T}$ as a condition to access this operation or, more generally, to activate/deactivate some function of the card.

This will be represented by $\mathrm{T} \Rightarrow \mathrm{F}$ as a time guarded command. 
It means that $\mathrm{F}$ can be used if and only if $\mathrm{T}=$ True, that is a time condition is verified within the card.

Example : The A function, which is an access control function to a card, is only permitted before a certain date. At that date the event END occurs. Then the security function will be :

$\mathrm{P}(\mathrm{END}) \Rightarrow \mathrm{A}$

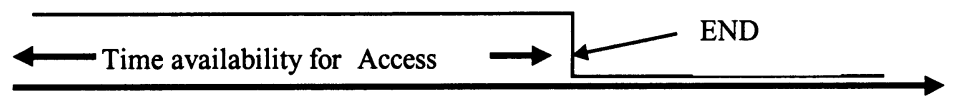

Figure 3. Exemple

It then becomes possible to organize the security scheme by producing the appropriate events and implementing the corresponding time guarded commands on the functions inside the card.

\subsection{Event possibilities in a smart card}

There are two possibilities to produce an event for a given device, namely a smart card, and these can be categorised as either External or Internal. From a security perspective the card can only guarantee the internal validity of the information. However external information can be compared to assist with security validation.

External sources : In the case where an event is initiated by another device, such as a terminal reader, and presented to the card then the card is only responsible for producing the internal time security function and to apply the time variables to any command or set of commands.

It is desirable that an external source will have to be authenticated in some way to prevent any fraudulent source replacing the correct one or detecting alterations in some way. It is the responsibility of the external device to provide the data associated with a particular event whatever that may be. The card just has to be prepared to receive the data. Practically, there are two possible sources of external time, the terminal which already includes a clock, or the use of a radio channel specialising in time broadcasting. Neither of them is considered as sufficiently trusted.

As a card is not active all the time, we must extend the definition of the BEFORE and the AFTER functions. Assuming that a card is only capable of taking an event into account when connected to an application (terminal), making it necessary to extend the definition of Past and Future to the presentations of the card.

There are many events which occur in real time but their implementation is postponed until the card is again connected to a card terminal. This is 
known as a postponed event $E^{\prime}$ for the card and is obtained from the real event $E$ and the next presentation $\operatorname{Pr}$ of the card by : $E^{\prime}=\operatorname{Pr}$ and $F(E)$. $E^{\prime}$ is the only event a card can acknowledge.

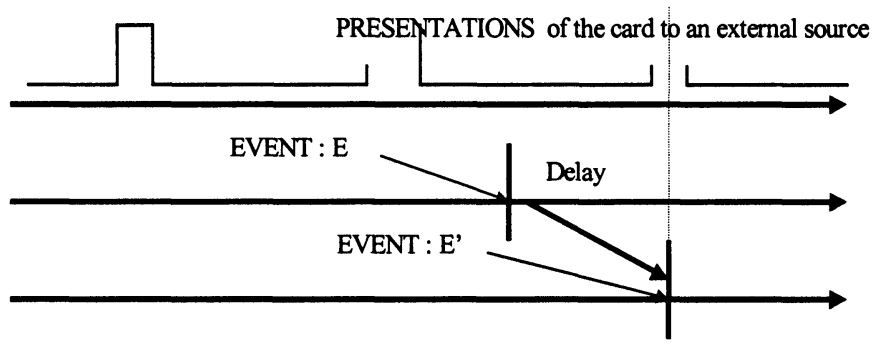

Figure 4. Presentation of the card to an external source

In this case $\mathrm{E}$ is created in the application before the card is presented to the terminal at E'. Using a postponed event $\mathrm{E}^{\prime}$ to replace the real event $\mathrm{E}$ usually does not create any extra problem as the card is totally idle between $\mathrm{E}$ and $\mathrm{E}^{\prime}$.

An example would be where the application updates the data, at say midnight, but the card connects to the system at 9am to use the updated data.

Internal sources : In this case, the card would be responsible for producing an event by itself and for its own purpose. The solution is more secure but, presently, the only available possibility is to create a program loop or any equivalent software delay by using the external clock generator. With presently available cards, this is only possible when the card is plugged into a terminal and cannot operate between two presentations so no internal time or date keeping is possible.

\section{PROPOSAL FOR AN INTERNAL TIME SOURCE.}

\subsection{Time Counter}

Although there are no common commercial smart cards with time clocks it is feasible to provide permanent power on a limited part of the card chip and hence it is possible to give the card an internal time keeper. This limited circuit would contain:

An oscillator.

A blind frequency divider to produce a 1 hertz (one second) clock 
A binary counter B to count as many seconds as necessary. With 27 bits, it is possible to count up to $134,217,727$ seconds which provides more than four years life for the card.

A thin battery to permanently deliver power to these components.

A brief technical analysis leads us to ignore the option of a quartz oscillator. Currently the quartz clock is a relatively large component and is not easily compatible with the thickness of the card. But moreover, a quartz clock is a separate component and that makes a physical attack easier. Even if the accuracy of time keeping is not as perfect as with a quartz, an RC oscillator with good temperature compensation will be preferred because it can be designed inside the chip at the time of manufacture and benefit from a tamper resistance level equivalent to the card itself.

The energy assessment depends on the way the battery is designed. One option is to recharge the battery at every connection but the connection time is short and some users can wait for a long time between two connections to a terminal device. A computation of what 36 flip-flops, designed with a very low power, low voltage technology shows that presently available thin film batteries can match both the limited available surface of the card and the desirable lifetime of the card, three or four years, for example.

\subsection{Limited Commands on the Card}

In the proposed model no SET or RESET commands are permitted on the counter. The clock starts counting from the moment the battery is connected during the manufacturing process. It never stops counting during the lifetime of the card ( which would be defined by the issuer as less than four years). Effectively the clock cannot be stopped or the time counter adjusted once it is initiated at manufacture. The unique permitted time command is GET TIME which allows a copy of the contents of the B counter to be moved to four one ( $8 \mathrm{bit}$ ) byte registers of the RAM. Then the current value of B is made accessible to the relevant programs or applications.

This feature presents two advantages:

The set of commands for time control is limited to a single instruction (GET TIME).

The internal time cannot be corrupted by any logical attack as it cannot be altered.

\subsection{Date Initialisation}

Both the functionality and the security options will be improved by storing an unalterable initialisation date. This date is likely to be located in the EEPROM storage of the card and 'fixed' at the time of manufacture $\left(D_{0}\right)$. 
The $D_{0}$ value provides a reference point of the date which is known to be correct for time management software. This date cannot be altered and is a known accurate and authenticated reference point of time for the card.

\section{THE TIME MANAGEMENT LAYER.}

At the personalization (card creation) stage, two elements of data are stored in the permanent memory: The current value of $B$, let us say $B_{0}$ (usually zero at manufacture) and the current value of the date and time $\mathrm{D}_{0}$. These values are fixed as the internal reference for the card. This data couple will be used as the time reference point for any future time calculations.

The clock counts from $\mathrm{B}_{0}$ and when the card is inserted in a terminal the GET-TIME hardware command is immediately and automatically executed providing $B_{t}$ the current value of the $B$ counter.

From $D_{0}$, and $B_{0}$ on one hand and $B_{t}$ on the other, the program will compute the present date $\left(D_{t}\right)$. An obvious opportunity exists to compare the date/time known to the card $D_{t}$ and the externally provided date from an application terminal $\mathrm{D}_{\mathrm{e}}$.

Actually two partly different presentations of the present date are made available to match with any application requirement. Every presentation of a date is composed of eight values.

Seconds : $\mathrm{S}=<0,59>$

Minutes : $\mathrm{M}=<0,59>$

Hours : $\mathrm{H}=<0,23>$

Days of the month : $\mathrm{DM}=\langle 1,31>$ or day of the week : $D W=\langle 1,7\rangle$

Month : $\mathrm{MO}=<1,12>$ or Week : $W=<1,52>$

Year : $\mathrm{Y}=<0,99>$ 


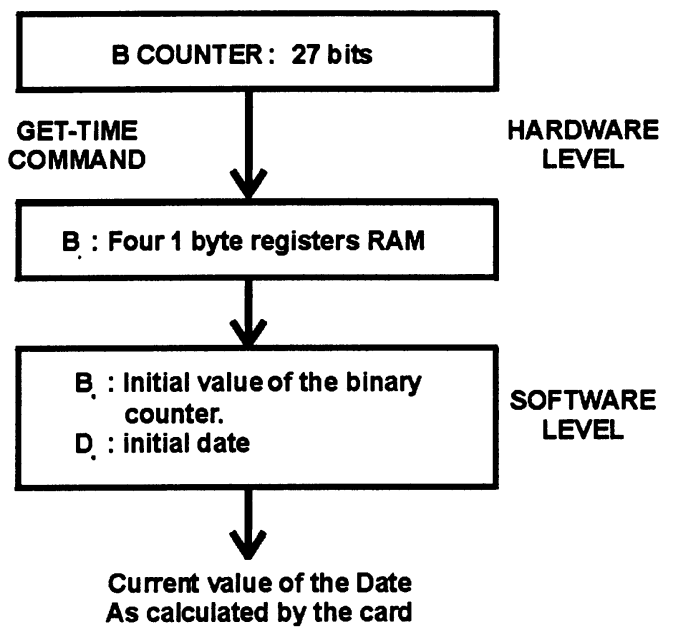

Figure 5. Transformation

Events can be:

- A complete date and time stamp: for example 12 of January 2001 at 06:32:27.

- The present time identified by NOW.

A time slice can be constructed in three different ways.

- As a function of one or more events.

Example : P(31, December, 1999); F(1, January, 1999) And P(31, December, 1999)

- A time slice issued from one or a few elements in the representations of a date.

12 of December 1999 (with a one day duration);

December 1999( with a 31 days duration).

The twentieth of the month for months: 2, 4, 6, 8, 10 and 12.

Day 3 of the week and year 2000 (every Wednesday of year 2000)

- A starting event plus a duration.

From 12 of December 1999 for 9 days.

Time slices may be combined by any AND, OR, NOT operator to produce a final description of the time condition to be used as a guarded command for any activity of the card. Finally a guarded command can be any description of a time function with one or more parts and possible cycles related to the clock of the card. The internal representation of a time slice is standardized according to the three basic type models 


\begin{tabular}{|l|l|l|}
\hline Type 1 & Date 1 & Date 2 \\
\hline Type 2 & Element of date & Values \\
\hline Type 3 & Date 1 & Duration \\
\hline
\end{tabular}

Figure 6. Time slices

Note that the model assumes the card will come into use after $1 / 1 / 2000$ consequently there are no dates in the form 19XX. Note also that the number of bits available for the year is only 4 providing for 16 years. It is assumed at that time improved technology will have allowed for more storage in the card. A date is represented by a 32 bit word in the card detailed in the following format:

\begin{tabular}{|c|c|c|c|c|c|c|}
\hline Type A & Second & Minute & Hour & $\begin{array}{c}\text { Day of } \\
\text { the month }\end{array}$ & Month & Year \\
\hline 2 bits = 00 & 6 bits & 6 bits & 5 bits & 5 bits & 4 bits & 4 bits \\
\hline Type B & Second & Minute & Hour & $\begin{array}{c}\text { Day of } \\
\text { the week }\end{array}$ & Week & Year \\
\hline 2Bits = 01 & 6 bits & 6 bits & 5 bits & $\begin{array}{c}3 \text { bits } \\
6 \text { bits }\end{array}$ & 4 bits \\
\hline
\end{tabular}

Figure 7. Format of a date

Example : 00(Type bits) $-00-00-13-12-08-(20) 02$ : is Type 1 format of the date August 12, 2002 at 1 PM

A duration is also represented by the same format with the same elements

The format for a cycle is quite different as it only refers to a unique element; the three bits of the second field identifies the element. The time slice goes from the initial value to the final value.

\begin{tabular}{|c|c|c|c|}
\hline Type C & $\begin{array}{c}\text { Element for } \\
\text { cycle }\end{array}$ & Initial value & Final value \\
\hline 2 bits $=10$ & 3 bits & 6 bits & 6 bits \\
\hline
\end{tabular}

Figure 8. Format for a cycle 
Example $:$ Type $=10$ with data $2-2-5$ points hours beginning at hour 2 to hour 5

Time slices that are created by the card designer are stored in a dedicated file of the operating system (Time Slices File) or TSF; every record of the file is given an index. This index is used to access the description or the time slice record.

\section{THE USE OF TIME GUARDED COMMANDS.}

An important aspect of the implementation is to decide where and how a time guard is used. By looking at the general architecture of the card, we can identify three sorts of objects that may be set under the authority of a time guarded command.

\subsection{Applications or users.}

Both of them are identified as various sets of external partners with a specific profile.

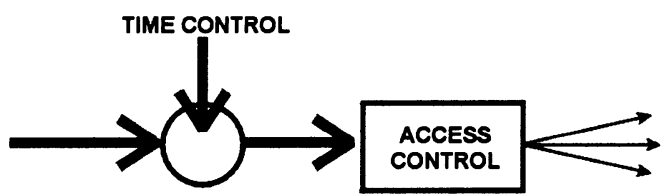

Figure 9. Control of applications or users

\subsection{The Instructions.}

A smart card only executes instructions communicated by the terminal. It is possible to relate every instruction to a temporal condition.

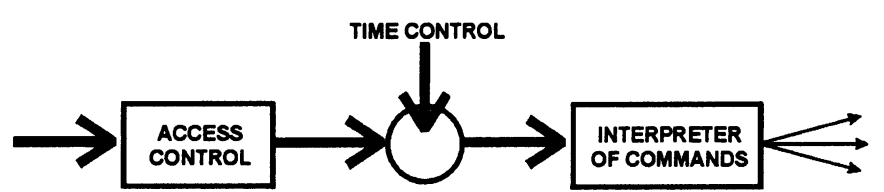

Figure 10. Control of instructions 


\subsection{Files or Tables}

The files (ISO 7816-4) or tables (ISO 7816-7) already have access which can be controlled by a secret key in memory and accessed only by the operating system. It is not then difficult to add in a similar fashion a guarded command as an extra security condition.

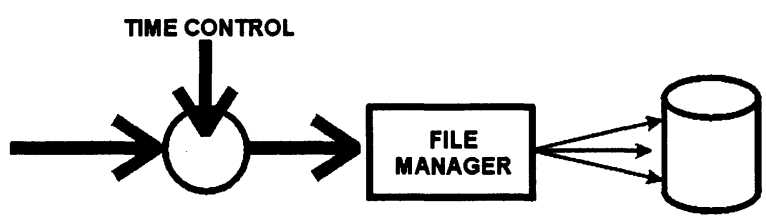

Figure 11. Control of files or tables

Actually, the decision on the best use of time control depends on the type of card and the application. To offer the application designer a wide spectrum of possibilities, it could be useful to mix these possibilities and to allow a variety of combinations of these criteria. However the following observations are made to help simplify the design for a possible implementation. First any time control is strongly related to what kind of services a user is permitted to obtain from the card. Then, at a fine grain analysis, the object that has to be controlled is expressed by the following sentence:

\section{$\mathrm{T} \Rightarrow$ USER-X asks to execute INSTRUCTION-Y on FILE-Z}

It is first possible to eliminate most of the possible instructions acting on stored files and to only keep the READ and WRITE ones. Any other instruction, such as CREATE, DELETE, COPY, ... are under the management level of the card and do need some form of time control. A multi-application card will certainly be accessible by many users for many different files; Then the link to optimize is the one between them.

It is proposed to use the Graham-Denning array model of security where the vertical entries are for users and the horizontal ones for files. Every box contains two guarded commands, one for the read instruction and the other one for the write instruction. A guarded command may be a logical expression combining any sort of time slice record.

For example : READ : T1 and T2 ; WRITE; T3 and T4 or T2 and not $T 1$.

This solution is very similar to the regular security model using keys which already exists in many smart cards. Such a choice does not require significant change to the basis of the operating system. But it is flexible enough to match with most of the application requirements. by

A general representation for a time security condition will be represented 
USER ID $\rightarrow$ Logical time function $(\mathrm{T} 1, \mathrm{~T} 2, \ldots . \mathrm{Tn}) \Rightarrow$ READ or WRITE the targeted FILE.

Of course this condition will very often be combined with a secret key condition.

\section{CONCLUSION.}

By adding a real time clock in a smart card, it becomes possible to take into account a large variety of time conditions and to significantly improve the level of security of the card and in turn the application. The prototype we propose is currently available as a software simulator. The main goal is to create a real version dependent on the possibility of using an efficient and cheap power supply for a lifetime or three or four years. In this case the card would contain the following services:

On line applications :

Watch-dog for a terminal or remote server response.

Limited time for PIN code presentation or between two presentations.

Reduction of the available time for an attack.

Off line applications :

Limited duration of a privilege

Minimum time between two sessions

Time or date control to access a function

Hourly, daily, weekly or monthly based cycle.

Postponed opening of a privilege

Periodic activities such as garbage collection

Deletion of ancient data.

Another problem to be solved is the accuracy of the clock. A possible solution is to reset the clock by a few minutes or seconds every time the card is plugged into a reader. The real time value will obviously not be changed but the software references (B0, D0) may be slightly shifted to take in account a difference between the time of the card and the time of the reader. However such an option opens up the opportunity to tamper the internal time.

If the real time clock is available, it also can be used for many other purposes; for example to produce well guaranteed time certificates or signatures. The fastest part of the clock can also be used to bring some programming facilities against time attacks by delivering elapsed times between two GET-TIME instructions.

But the most important condition in making such a card available at the commercial level is to identify a real need for more security by time control. 
Potentially, it is likely that many existing or future applications can benefit from such a feature.

\section{BIBLIOGRAPHY}

ANALYSES \& SYNTHESIS - http://www.cardshow.com, February 24, 1999.

BALME, L., SILVY, C. - Project Smart Power card, Activity report, Laboratorie TIMA, Techniques de l'Informatique et de la Microelectronique pour l'Architecture d'ordinateurs, University de Grenoble, 1992.

BESTOUGEFF H., LIGHOZAT G. - Time treatment software - from linguistic to artificial intellect, Masson, Paris Milan Barcelona Mexico, 1989.

CORDONNIER V., NEMCHENKO V., KRIVOULYA F., NEMCHENKO S. - Smart cards application in the information space of modern society, Radioelectronika $i$ informatika, Kharkiv, Ukraine, 2(3), 125 - 127, 1998

CORDONNIER V., WATSON A.C. - "Access Control Determination of Multi-Application Smartcards Using a Security Index", Third Australasian Security Research Symposium, Queensland University of Technology, July, 1997

ELEA CARD WARE - http://www.eleacard.com/fr_acc.htm, The smart cards access control, March 99.

GRAHAM-DENNING -

http;//www.cs.nps.navy.mil/curricula/tracks/security/notes/chap08_9.html\#HEADING8, The Graham-Denning Model

TONDA BENES - http://www.kolej.mff.cuni.cz/prednes/oi0a.html, Security in the Operating Systems, KSI MFF UK Praha, 1996.

VANDEWALLE J.-J. - Smart card course, IUT « A » Lille 1, Informatics Department.

ZAVALEEV V. - Smart card as the payment tool, Information technologies centre, http://www.citforum.ru/marketing/articles/art_8.shtml 